\title{
Des femmes en bibliothèque au temps du Kaiser : deux expériences strasbourgeoises
}

\section{Daniel Bornemann}

\section{OpenEdition}

1 Journals

Édition électronique

URL : http://journals.openedition.org/rbnu/1196

DOI : 10.4000/rbnu.1196

ISSN : 2679-6104

Éditeur

Bibliothèque nationale et universitaire de Strasbourg

\section{Édition imprimée}

Date de publication : 1 novembre 2016

Pagination : 56-61

ISBN : 9782859230630

ISSN : 2109-2761

\section{Référence électronique}

Daniel Bornemann, «Des femmes en bibliothèque au temps du Kaiser : deux expériences strasbourgeoises », La Revue de la BNU [En ligne], 14 | 2016, mis en ligne le 01 janvier 2020, consulté le 11 décembre 2020. URL : http://journals.openedition.org/rbnu/1196 ; DOI : https://doi.org/10.4000/ rbnu. 1196

\section{(c) (†)(2)}

La Revue de la BNU est mise à disposition selon les termes de la Licence Creative Commons Attribution - Pas d'Utilisation Commerciale - Partage dans les Mêmes Conditions 4.0 International. 


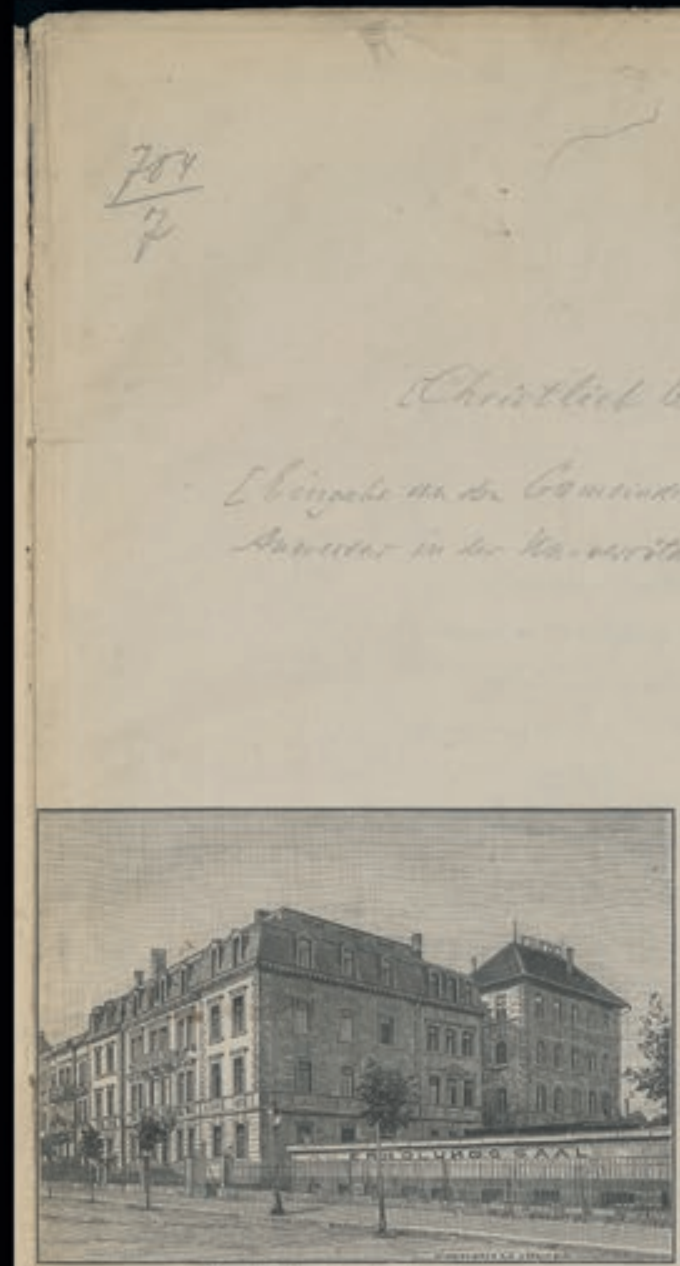

Dr. Hottingers Anweses.

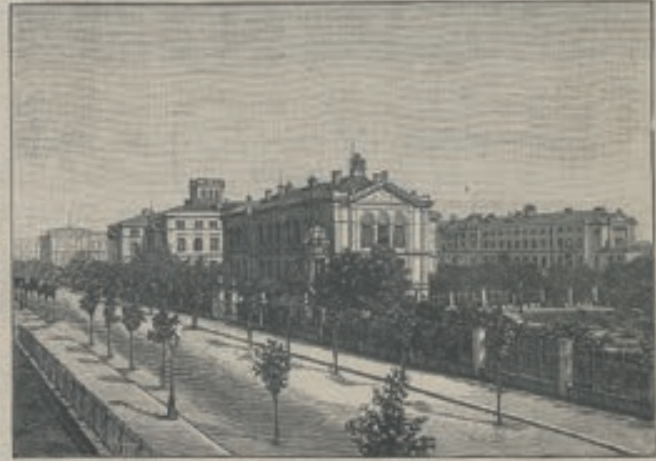

Blick von Dr. Hottingers Anwenen anf einige Universitiitsinstitate. 


\section{DES FEMMES EN BIBLIOTHÈQUE AU TEMPS DU KAISER : deux expériences strasbourgeoises}

A

u cours d'un travail sur les archives institutionnelles de la BNU pendant la période $1871^{-}$ 1919, qui a pour objectif de rendre accessible ce corpus à la communauté scientifique en le cataloguant sur l'outil en ligne Calames ${ }^{1}$, plusieurs dossiers concernant la formation des personnels féminin $s^{2}$ ont été examinés. Il semble opportun, dans le cadre du dossier thématique de ce numéro de La Revue, d'attirer l'attention sur l'existence de ces archives, dans l'espoir que des chercheurs voudront les étudier plus à fond. Nous n'en proposons ici qu'une brève et succincte description.

Il faut bien distinguer deux phases, sans grand lien entre elles, dans l'émergence des formations pour demoiselles aux métiers des bibliothèques, à Strasbourg au temps du Reichsland d'Alsace-Lorraine. La première est de l'ordre de l'initiative privée, mais conduite par un personnel de la bibliothèque en dehors de son service : le Dr. Christlieb Gotthold Hottinger (1848-1914), à partir de 1888-1889. La seconde phase est la mise en place par la direction de la KULB 3 à partir de 1907 , en accord avec d'autres instances locales, d'un projet de diplôme de femme-bibliothécaire qui trouvera une application effective de 1913 jusqu'en juillet 1918.

La première de ces démarches est décrite dans le dossier personnel de Hottinger, en tant qu'employé de la KULB où il était " custos ", autrement dit conservateur, en charge de la théologie et de la philosophie. Ce dossier, plutôt abondant pour un dossier personnel, compte 276 feuillets. Outre les documents relatifs au déroulement de sa carrière, il contient aussi, mais de manière indirecte, un grand nombre d'informations sur l'établissement d'enseignement destiné aux femmes qu'Hottinger a mis sur pied. Témoignant d'une attention quelque peu suspi- cieuse, l'administration de la bibliothèque a réuni des documents de diverses natures qui permettent de s'en faire une idée plus ou moins claire. Même au-delà du départ en retraite anticipée de l'employé (pour raison de santé) en 1894, le dossier continue à en réunir, ce qui est pour le moins inhabituel. En effet, à partir de cette retraite anticipée, le projet d'Hottinger prend un certain essor durant quelques années, d'abord à Strasbourg puis, à partir de 1899, à Berlin-Südende. Même à partir de son départ pour la capitale de l'Empire, des informations sur son activité sont encore collectées par l'administration de la KULB et enregistrées dans son dossier personnel.

Qu'en est-il de cet établissement ? Il apparaît comme une institution multifonctionnelle, destinée aux femmes. Il contenait une école de caractère généraliste pour les jeunes femmes, avec un volet d'enseignement relatif aux métiers des bibliothèques et de la librairie, mais aussi un " home " pour les femmes (pour les femmes seules ou en difficulté ?), une salle d'exposition et un " lieu de convivialité " ${ }^{4}$. L'enseignement, tel qu'annoncé par la brochure concernant l'établissement, n'est pas entièrement consacré à la bibliothéconomie. Des cours d'histoire, de géographie, d'écriture entre autres, forment un cursus apparemment assez complet. Mais des cours sur le commerce du livre, sur la reliure, sur l'imprimerie ou encore sur la gravure sur bois sont également dispensés. L'établissement de Berlin-Südende est, lui, clairement orienté vers le travail en bibliothèque, montrant que le projet de Hottinger s'est concentré sur ces métiers. Il est d'ailleurs l'auteur d'un écrit intitulé Die Bibliothekarin (La bibliothécaire), daté de 1903, qui ne compte cependant que quatre pages et date de cette période berlinoise.

Cela étant dit, le dossier offre un regard biaisé sur les initiatives de Hottinger, et les données complètes 
sur l'établissement devront être recherchées aussi dans d'autres services d'archives (Archives départementales $\mathrm{du}$ Bas-Rhin et archives berlinoises en particulier). Mais les informations présentes valent le détour, car d'une certaine manière Hottinger y apparaît parfois comme un " chevalier d'industrie ", en tout cas dans l'optique de certains de ses collègues. N'a-t-il pas formé plusieurs projets colossaux dont une " biobibliographie générale "(Bio-Icono-Bibliographia universalis, rien moins que cela !) sur souscription, ou encore un " nouveau Konversationslexikon, le plus complet et le moins cher du monde " ? Il a aussi proposé plus modestement à sa hiérarchie la réalisation d'un catalogue imprimé de la collection des alsatiques de la KULB ${ }^{5}$. Mais le succès ne lui a pas souri. Peut-être y eut-il même quelques malversations en ce qui concerne des souscriptions non honorées. Hottinger fut tout de même décoré par l'attribution de la croix de chevalier de $1^{\text {ìre }}$ classe du Grand-duché de Bade et par celle, en 1888, de l'ordre du Lion de Zähringen, ce qui laisse penser qu'il était respecté et reconnu par son entourage professionnel.

C'est en employant les élèves de son institution à la constitution de ces ouvrages que Hottinger pensait pouvoir en arriver à bout. Des échantillons de ces travaux préparatoires se trouvent dans le dossier, et pour se procurer la bienveillance de ses collègues de la KULB, il faisait rédiger de manière soignée et illustrée des fiches bio-bibliographiques concernant ces derniers et ses supérieurs hiérarchiques (Karl August Barack, Julius Euting par exemple). L'enseignement formait donc des "documentalistes " capables d'effectuer des recherches bio-bibliographiques et de mettre en forme les résultats de celles-ci. La section alsatique de la BNU a conservé aujourd'hui des brochures, dépliants et quelques-unes des œuvres de circonstance produites par Hottinger. Consacrant tel livret à la journée de Sedan (le "Sedantag " commémorant la victoire allemande de 1870), tel autre à l'empereur Guillaume Ir ou à d'autres faits patriotiques, il cherchait visiblement à obtenir la reconnaissance et l'assistance des autorités au pouvoir afin de mener à bien ses grands projets.

La production écrite de Hottinger n'est pas du tout négligeable, en particulier pour l'histoire de la bibliothéconomie allemande et alsacienne. Elle est bien présente dans les fonds alsatiques de la BNU, notamment les volumes de ce qu'il nommait les "orbis pictus " où de nombreux sujets étaient traités " par les mots et les images ", " in Wort und Bild ". Par ailleurs, de nombreuses coupures de presse concernant ses projets stras- bourgeois ou berlinois se trouvent consignées dans son dossier personnel, et témoignent du regard parfois ironique porté par l'opinion publique strasbourgeoise sur ses entreprises.

Le second moment de la mise en place d'un enseignement bibliothéconomique pour les demoiselles ${ }^{6}$ à Strasbourg forme un ensemble de dossiers d'archives beaucoup plus structuré. C'est en effet l'administration de la KULB qui est cette fois à l'initiative du projet. Il s'agit d'instaurer une formation pour bibliothécaires féminines, une "Bibliothekarinnenschule ». Cette idée naît entre 1907 et 1911, sous l'influence d'un écrit de l'Association professionnelle des femmes bibliothécaires (Vereinigung bibliothekarisch arbeitender Frauen) publié à Berlin en 1907, et qui est présent dans le dossier d'archives. L'idée de l'administration de la bibliothèque est de créer une commission d'examen permettant le recrutement de personnels féminins formés et choisis selon des critères précis. S'appuyant sur l'expérience d'un enseignant badois, Oskar Meyer (d'Ohlsbach près d'Offenbourg), et sur des informations provenant de l'école de Südende (celle de C. G. Hottinger), elle institue une école par un décret du 15 juin 1912 publié au Journal officiel d'Alsace-Lorraine'. L'" Oberbibliothekar " et responsable des alsatiques à la KULB Ernst Marckwald, mais aussi le libraire Trübner et Samuel Landauer (également Oberbibliothekar, chargé des secteurs de la médecine et des sciences naturelles) sont chargés de constituer un jury et d'élaborer le programme des concours. L'école proprement dite se tient dans un institut de formation strasbourgeois, la Köbig'sche höhere Mädschenschule, située Manteuffelstrasse 24 (l'actuelle rue du Maréchal Foch).

Le contenu de l'enseignement et les modalités des examens, détaillés dans le dossier, sont en constante évolution. Des avis critiques sur la méthode ou sur les contenus sont collectés, et les sentiments mêlés des examinateurs sont également exprimés à l'occasion. Tout ne va pas pour le mieux dans cette formation, et ce qui paraît intéressant dans ces dossiers, comme d'ailleurs dans le premier ensemble, c'est de percevoir les difficultés rencontrées, les pesanteurs ressenties, les doutes et les regrets parfois exprimés par les personnes qui encadrent la formation. Les rapports relatifs aux sessions de 1913, 1914, 1916, 1917 et 1918 y sont présents (il n'y eut pas de session en 1915).

D'autres dossiers viennent compléter l'ensemble : ils contiennent les nombreux travaux d'examens réalisés par les candidates au diplôme. Il y a d'une part un dossier concernant celles qui ont postulé pour un emploi 


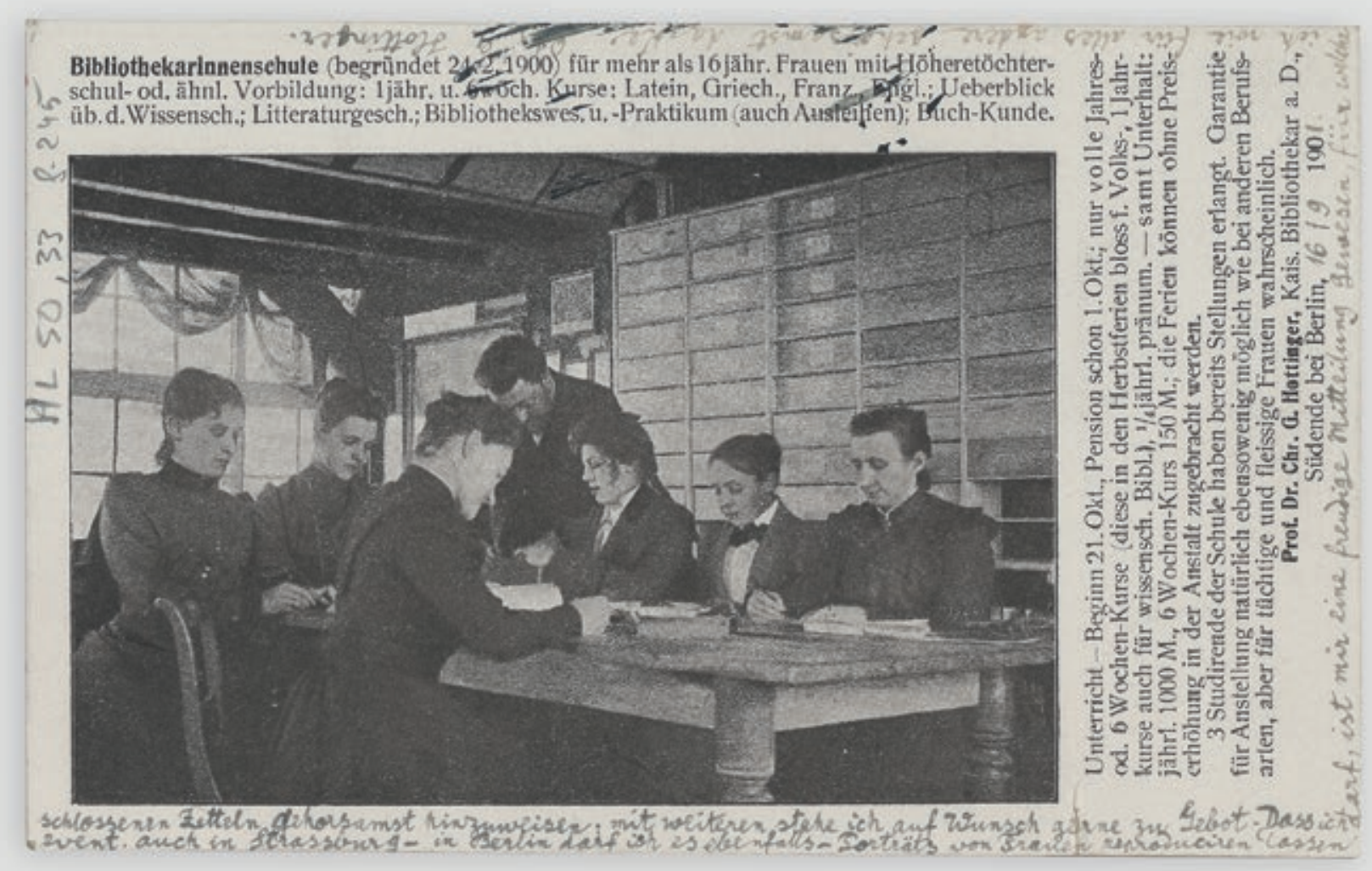

L'établissement de Berlin-Südende. Carte postale de 1900-1901 mentionnant le programme et les contenus de l'enseignement (coll. BNU) 


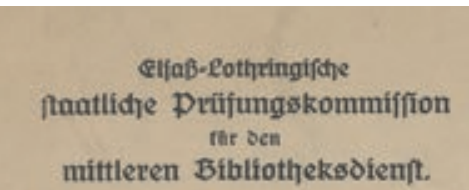

\section{Zeunnis.}

Dor oer prafungskommiffion fute oen mittleren Btbliotheksolenf an miffenfthaftlidjen Bibliotheken fowie für oen Dienf an Dolkabibliotheken uno verwanðten Jnftituten hat

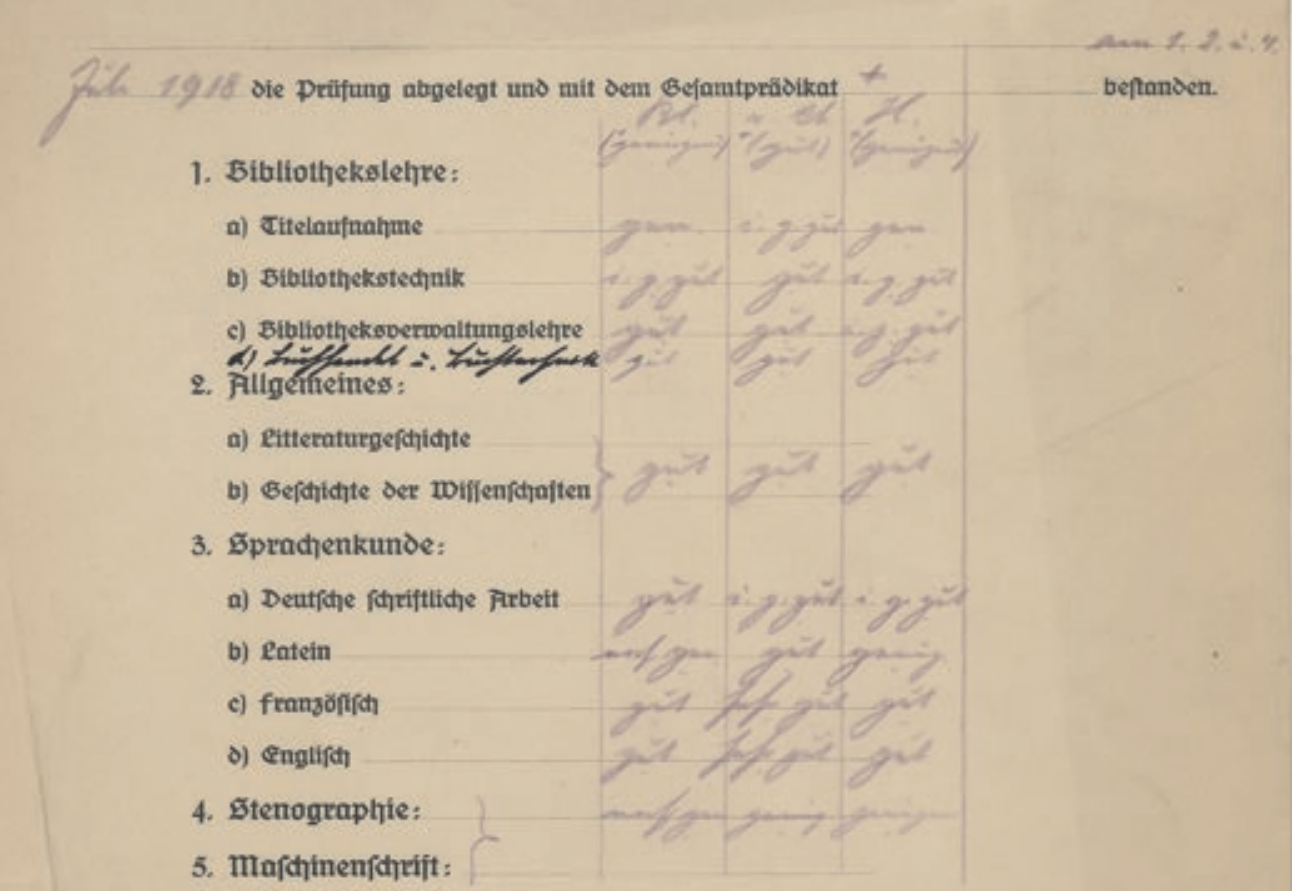

Befondere Bemerkungen:

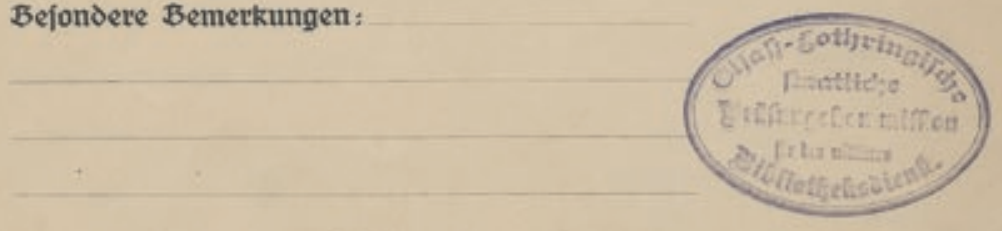

Strubburg i \&., den of $22<02$

Der Dorfibende der Prüfungskommiffion:

L.s.

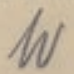


à la KULB ${ }^{8}$ dans les années 1913 et 1914. On y trouve les pièces originales des épreuves proposées à ces candidates qui sont : une rédaction sur un sujet donné, une prise de notes en sténographie, l'écriture (calligraphique) de nombreuses fiches bibliographiques, la rédaction et la mise en forme de lettres officielles, des pages de dactylographie, le curriculum vitae de chacune d'elles et les appréciations des examinateurs. D'autre part, on possède les dossiers de toutes les jeunes femmes qui ont candidaté 9 et participé à ces formations, sans être finalement employées à la KULB. Ces dossiers personnels contiennent également toutes les pièces attendues : les lettres de candidature, les curriculum vitae, les travaux réalisés au cours des examens. Cette série homogène de données sur les candidates à un examen professionnel bibliothéconomique devrait pouvoir faire l'objet d'une étude circonstanciée, et mettre la lumière sur un chapitre somme toute peu connu de l'histoire de la formation professionnelle féminine.

On voit en tout cas, par le contenu des examens, que c'est au " service moyen " (le " mittlerer Dienst " allemand) que ces femmes étaient destinées professionnellement. Il s'agit bien de fonctions subalternes et non de responsabilité d'établissements. Ces messieurs ont dû beaucoup discuter sur les possibilités de carrières de ces personnes $\mathrm{du}$ " sexe faible ». Leur intégration dans les emplois de haut niveau n'allait pas de soi dans la mentalité d'alors et n'était visiblement pas souhaitée par l'administration. Après le retour de l'Alsace à la France, on allait nommer les personnels ainsi recrutés les "sous-bibliothécaires ${ }^{10}$. L'objectif de ces diplômes était d'orienter les lauréates vers des emplois dans les bibliothèques populaires et dans les cercles de lecture ("Volksbibliotheken und Lesehallen "), plus que dans les bibliothèques d'une certaine importance. Mais plusieurs personnels féminins furent recrutés à l'issue de cette formation par la KULB. Rappelons au passage qu'en Allemagne, et ce jusqu'aux années 1950, il était mal vu de poursuivre une carrière professionnelle à partir du moment où l'on était mariée et surtout mère de famille. Aussi toutes ces femmes sontelles bien officiellement qualifiées de " demoiselles".

Un tel ensemble, qui trouvera peut-être d'importants compléments dans d'autres services d'archives alsaciens, allemands, du pays de Bade ou de Berlin, doit pouvoir apporter des éléments intéressants sur la question de la présence des femmes dans les bibliothèques, ici du point de vue de la profession de bibliothécaire. Le contexte est celui de l'Empire allemand de la fin du $19^{e}$ siècle, jusqu'à la fin de la Première Guerre mondiale. C'est l'époque où se pose la question de l'intégration professionnelle des femmes dans les sociétés occidentales. Des recherches sur les éventuelles carrières ultérieures de ces candidates (ou lauréates) en Alsace ou en Allemagne, après 1918, constitueraient autant de perspectives stimulantes. Ce sont en tout cas des dossiers pleins de vie(s), qui possèdent le don de l'éternelle jeunesse, et où l'on peut même croiser parfois un brin de sourire.

\section{Daniel Bornemann}

\section{Notes}

1 - Calames (www.calames.abes.fr) est le catalogue en ligne des archives et des manuscrits de l'enseignement supérieur, géré par l'ABES, Agence bibliographique de l'enseignement supérieur. Tous les fonds manuscrits de la BNU y sont décrits. Leur encodage en EAD est effectué avec le soutien de l'ABES.

2 - Le dossier Hottinger a pour cote $\mathrm{AL}$ 50,33. Son titre : Acta personalia betreffend den Bibliothekar Dr. Hottinger.

3 - KULB : Kaiserliche Universitäts- und Landesbibliothek zu Strassburg, nom de la BNU jusqu'en 1918.

4- Son nom : « Hottinger's Frauenschule, Frauenheim, Ausstellung und Erholungsabende ». La dénomination " Hottingers Garten » ou " jardins Hottinger » existe aussi. Ce complexe était situé entre la rue de l'Université et le boulevard de la Victoire (à l'époque Nikolaus Ring). L'institution est décrite dans la brochure Chr. G. Hottinger's 'Frauenschule', 'Frauenheim','Ausstellung' und 'Erholungsabende', Strassburg im Elsass, parue en 1887.

5 - Celui-ci sera réalisé et imprimé plus tard, à partir de 1908, grâce à la fondation de la famille de Gustav Mühl, par Ernst Marckwald et Ferdinand Mentz.

6 - Les dossiers ont pour cotes $\mathrm{AL}$ 50,14 et $\mathrm{Al}$ 50,15. Le premier contient les pièces relatives à la fondation et au fonctionnement de cet enseignement ; le second les travaux d'examens. Pour quelques références bibliographiques d'époque sur la fondation de l'école, voir Henri Dubled, Histoire de la Bibliothèque nationale et universitaire de Strasbourg, Strasbourg, $2^{\mathrm{e}}$ éd. 1973.

7 - Zentral-und Bezirks-Amtsblatt für Elsass-Lothringen du 15 juin 1912, $\mathrm{n}^{\circ} 24$, p. 101-103

8 - Cote du dossier AL 50,16 (8o f.). Il faut y ajouter le AL 50,17 bis.

9 - Nous en avons décompté 29, sans garantie d'exhaustivité. Les dossiers d'archives les concernant occupent les cotes $\mathrm{AL}$ 50,85 à AL 50,119. Certains de ces dossiers concernent des personnes qui vivaient encore ily a moins de cinquante ans.

10 - Voir à ce sujet l'article de Julien Gueslin, p. 96 